\title{
The Role of Non-linearity for Evolved Multifunctional Robot Behavior
}

\author{
Martin Hülse, Steffen Wischmann, and Frank Pasemann \\ Fraunhofer Institute, Autonomous Intelligent Systems, \\ 53754 Sankt Augustin, Germany \\ \{martin.huelse, steffen.wischmann, frank.pasemann\}@ais.fraunhofer.de \\ http://www.ais.fraunhofer.de/ INDY
}

\begin{abstract}
In this paper the role of non-linear control structures for the development of multifunctional robot behavior in a self-organized way is discussed. This discussion is based on experiments where combinations of two behavioral tasks are incrementally evolved. The evolutionary experiments develop recurrent neural networks of general type in a systematically way. The resulting networks are investigated according to the underlying structure-function relations. These investigations point to necessary properties providing multifunctionality, scalability, and openended evolutionary strategies in Evolutionary Robotics.
\end{abstract}

\section{Introduction}

Evolutionary robotics (ER) as the study and development of behavioral control for autonomous robots through self-organizing processes based on artificial evolution is a widely accepted approach [10/14. With respect to natural evolution and simplest forms of natural life there are many researches criticizing the dissatisfying outcomes of current work in ER [415]. In [5] it is claimed that open-ended evolutionary processes are necessary to overcome crucial limitations of current ER models and to generate more complex and interesting results.

However ER models providing open-ended evolutionary processes are implemented, the agents must be incrementally evolved. With respect to behavioral control this means control structures must facilitate incremental evolution. Such an approach should also cope with the scalability problem of ER models in general 1134 .

The crux of incremental control structure evolution is the integration of new behavioral functionality without loosing existing capabilities. In this paper we propose an approach to make this problem more tractable. We present incrementally evolved control structures which are systematically investigate to study the underlying dynamical properties and control principles providing (1) coordination of different behavioral tasks, and (2) the development of multifunctionality. In 2 it is claimed that a serious and systematical analysis of concrete examples of evolved agents are the prerequisite for dynamical explanation and "abstracting" general principles" of situated autonomous agents. Therefore we present robotic tasks which at first might seem rather simple, but this simplicity allows 
minimal systems which are exactly what we need to give a detailed description of the effects of the purposed incremental evolutionary approach on the dynamical properties of already existing control structures with innate basic functionalities. Based on these results we discuss the role of non-linearity for (1) open-ended evolutionary processes and (2) the development of multifunctionality in a self-organized way.

\section{Setup}

We present experiments which systematically apply two methods of incremental evolution of recurrent neural networks (RNN), also referred to as neuro-modules: (1) expansion method and (2) fusion method [6. Each method is realized by a restrictive and semi-restrictive technique. Restrictive means that neither already existing structural elements (hidden neurons and synapses) nor parameters (bias and weight terms) of the initial basic building modules can be changed. hereas, semi-restrictive means that parameters can be changed, while the structure remain fixed [6], too.

Expansion and fusion methods are realized with an evolutionary algorithm, the $E N S^{3}$-algorithm (described in 6[13]). Using a standard additive neuron model with sigmoidal transfer function $\sigma(x)$ and time discrete dynamics the $E N S^{3}$-algorithm evolves neural structures and optimizes the corresponding parameters at the same time. Besides from a task specific input-output structure, the neuron type, and the constraint that input neurons have only outgoing weights, nothing else is determined. Therefore, any kind of recurrent neural connections, like self-connections and loops can emerge during the evolutionary process.

As an incrementally evolved robot task, we chose a reactive light seeking behavior. Light seeking includes the coordination of a positive and negative tropism - phototaxis and obstacle avoidance. In the following light seeking behavior means that a robot has to follow a light source and has to stop in front of it while it is avoiding collision with any objects in its environment. For these studies the Khepera robot 9 and a 2-dimensional simulation software 8$]$ is used. Note, that all evolution experiments and analysis are done in simulation but all resulting controllers were tested on the physical hardware as well to approve that the observed behavior in real world is qualitatively the same as in simulation.

\section{Experiments}

The Khepera robot is driven by two DC-motors (control signals $m_{l}, m_{r}$ ), which are able to move the left and right wheel forward (positive signals) and backward (negative signals). The sensor data of the Khepera are delivered by its eight infrared sensors. They can be executed in two modes, measuring light intensity (sensor values $l_{0}, l_{1}, \ldots l_{7}$ ) and distances to obstacles (sensor values $d_{0}, d_{1}, \ldots, d_{7}$ ). The sensor values $l_{n}$ and $d_{n}$ are mapped into the closed interval $[0.0 ; 1.0]$. For the light sensors, values $l_{n}=0.0$ refers to darkness and $l_{n}=1.0$ to the maximal 
measurable light intensity. The proximity values $d_{n}$ are zero if no obstacle is detected and value 1.0 represents a collision. In all presented experiments the sensor values $d_{n}$ and $l_{n}$ are summarized as follows:

$$
\begin{array}{cc}
i_{1}:=\frac{1}{3}\left(d_{0}+d_{1}+d_{2}\right), & i_{2}:=\frac{1}{3}\left(d_{2}+d_{3}+d_{4}\right), \\
i_{3}:=\frac{1}{2}\left(l_{0}+l_{1}\right), \quad i_{4}:=\frac{1}{2}\left(l_{2}+l_{3}\right), & i_{5}:=\frac{1}{2}\left(l_{4}+l_{5}\right), \quad i_{6}:=\frac{1}{2}\left(l_{6}+l_{7}\right) .
\end{array}
$$

Where $i_{1}$ and $i_{2}$ represents the distance to obstacles at the robot's left and right side. The values $i_{3}$ and $i_{5}$ indicates the light intensity at the left and the right, $i_{4}$ the intensity at the front, and $i_{6}$ at the rear. According to this setup the inputoutput structure of the neuro-modules that were evolved for the light seeking task is represented by six input neurons $\left(I_{1}, I_{2}, \ldots I_{6}\right)$ and two output neurons $\left(O_{1}\right.$ and $\left.O_{2}\right)$. The values $i_{1}, \ldots, i_{6}$ are the inputs of the corresponding input neurons $I_{1}, \ldots, I_{6}$ of the neuro-module. Since input neurons are only used as buffers the values $i_{n}$ can also be seen as the outputs of the corresponding input neurons $I_{n}$. As transfer function we applied $\sigma(x):=\frac{1}{1+e^{-x}}$, the standard sigmoid. According to the problem of handicapped navigation possibilities with only positive control signals a special post-processing is implemented. We functionally decompose the two output neurons. The left output neuron $\mathrm{O}_{1}$ controls the speed and $\mathrm{O}_{2}$ the turning angle of the robot's movement. This is formalized as follows:

$$
\begin{aligned}
& m_{l}:=\left\lfloor\left(5.0 \cdot\left(o_{1}-\left(2 \cdot o_{2}-1.0\right)\right)\right)+0.5\right\rfloor, \\
& m_{r}:=\left\lfloor\left(5.0 \cdot\left(o_{1}+\left(2 \cdot o_{2}-1.0\right)\right)\right)+0.5\right\rfloor .
\end{aligned}
$$

In such a way we get positive and negative integer values, used as motor control signals for the Khepera robot, simulated as well as real.

\subsection{Basic Building Modules}

For the following experiments we used two basic building modules. The neural structure of module $\mathcal{G}_{\mathcal{O}}$, solving an obstacle avoidance task, and its resulting behavior in a simulated environment is show in Fig. 1 (a). This module has an even 2-ring between $\mathrm{O}_{1}$ and $\mathrm{O}_{2}$. Its weight configuration is critical and therefore hysteresis effects can be expected. A robot controlled by this module generates a straight forward movement, if no obstacle is detected. The robot is able to escape from dead-ends and sharp corners.

Fig. 1 (b) shows the second basic building module $\mathcal{G}_{\mathcal{L}}$, performing a positive phototropism. Module $\mathcal{G}_{\mathcal{L}}$ is basically feedforward organized. Hence, this module can only provide fixpoint attractors. The resulting robot behavior shows a strong drive to the right, if no light is detected. The drive to the right is forced by the bias term 0.2 of $\mathrm{O}_{2}$. The bias term causes an output value larger than 0.5 that generates a turning angle unequal zero. If the robot detects light it moves straight to it and stops in front of it. 
(a)

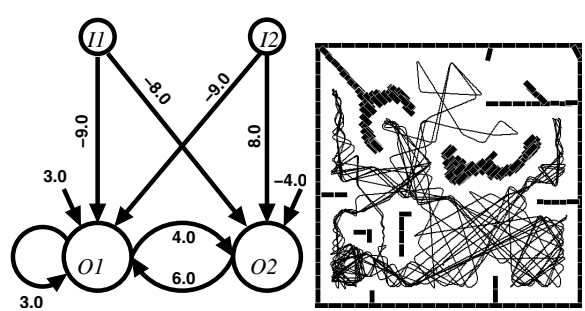

(b)

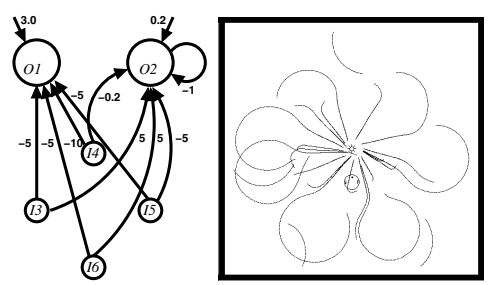

Fig. 1. (a) Neuro-module $\mathcal{G}_{\mathcal{O}}$ solving an obstacle avoidance task, (b) neuro-module $\mathcal{G}_{\mathcal{L}}$ performing a positive phototropism, and the resulting robot behavior in simulation

\subsection{Expansion of a Basic Module}

Two neuro-modules resulting from the expansion experiments are shown in Fig. 2] Neuro-module $\mathcal{G}_{\mathcal{O}} \Rightarrow \mathcal{L}$ is one outcome of the restrictive and $\mathcal{G}_{\mathcal{O}} \rightarrow \mathcal{L}$ one of the semi-restrictive expansion experiments.

Aside from the undercritical self-connection of $\mathrm{O}_{2}$ the new structural elements of $\mathcal{G}_{\mathcal{O}} \Rightarrow \mathcal{L}$ are only feedforward organized (Fig $2(\mathrm{a})$ ). These new connections are coming from the input neurons delivering light sensor data. With respect to the feedforward organization of the new connections one can not expect additional non-trivial dynamical effects. Robots controlled by this module show a drive to the right, if no obstacle and light is detected. If a light source is detected, the robot orients to it and comes to a halt in front of it. It has not lost its capability to escape from dead-ends (Fig 2(a)).

Considering the semi-restricted evolved neuro-module $\mathcal{G}_{\mathcal{O} \rightarrow \mathcal{L}}$ one can find only three new connections (Fig 2(b)). Like in module $\mathcal{G}_{\mathcal{O}} \Rightarrow \mathcal{L}$ all the new connections come from the input neurons delivering the light sensor data and are feedforward organized. However, the weights of the initial structure have strik-

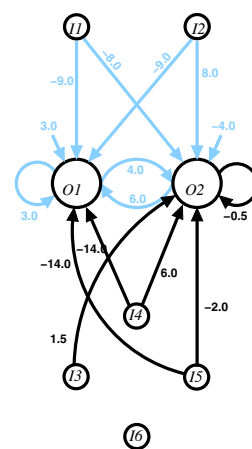

(a)

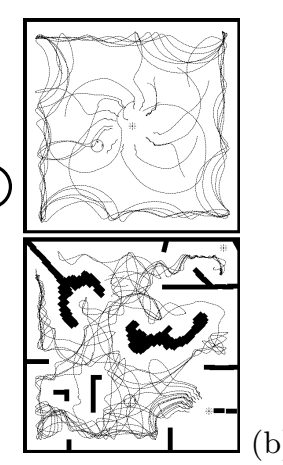

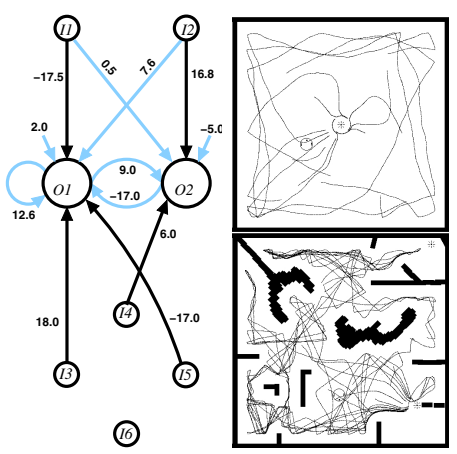

(1)

Fig. 2. Neuro-module $\mathcal{G}_{\mathcal{O} \Rightarrow \mathcal{L}}$ resulting from (a) the restrictive module expansion, (b) neuro-module $\mathcal{G}_{\mathcal{O} \rightarrow \mathcal{L}}$ resulting from the semi-restrictive module expansion, and the resulting light seeking behavior. The grey color indicates the unchanged elements. 
ingly changed. The self-connection of $O_{1}$ has become critical and it is now able to generate a hysteresis effect. Furthermore the 2-ring between $O_{1}$ and $O_{2}$ has become odd. This 2-ring can generate periodic and chaotic attractors [11. If the robot detects no obstacle and no light, its resulting behavior is characterized by irregular and slight drives to the left as well as to the right (Fig. 2(b)). The semi-restrictive evolved module $\mathcal{G}_{\mathcal{O} \rightarrow \mathcal{L}}$ can also escape from dead-ends as well as it comes to a halt in front of a light source.

\subsection{Fusion of the Two Basic Modules}

The initial structures of the following fusion experiments include the two modules $\mathcal{G}_{\mathcal{O}}$ and $\mathcal{G}_{\mathcal{L}}$. The output neurons of both modules become hidden neurons $\left(H_{1, \ldots 4}\right)$ of the initial structure. During the evolutionary process the insertion of new connections coming from the input neurons was not allowed. This guarantees that no structural elements emerge, which could exclude the basic modules. Figure 3 shows two examples of resulting neuro-modules, restrictive $\left(\mathcal{G}_{\mathcal{O}} \Leftrightarrow \mathcal{L}\right)$ and
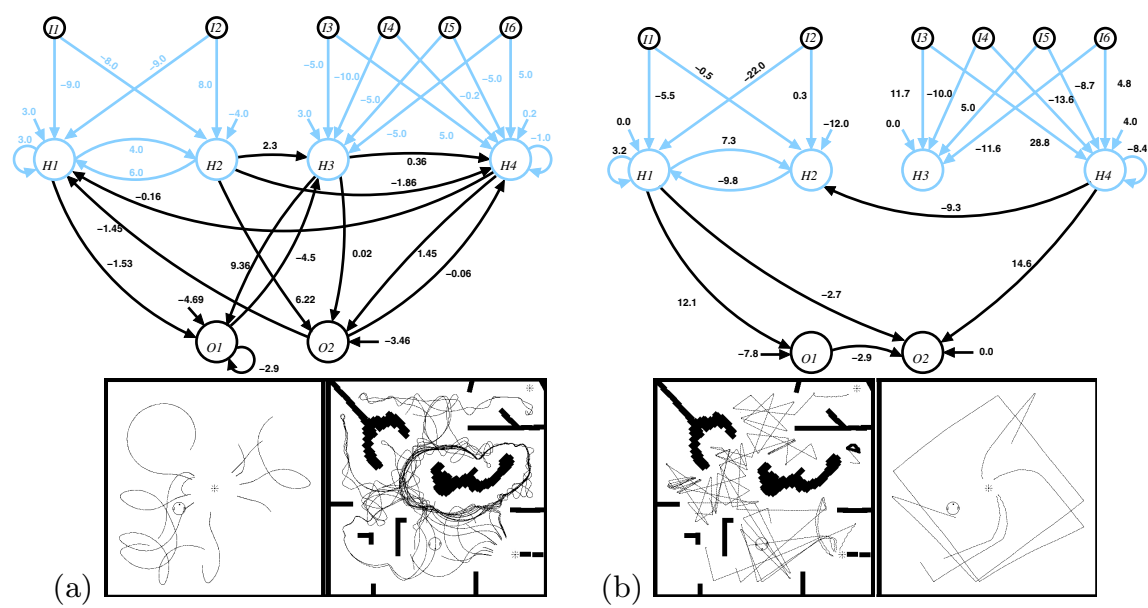

Fig. 3. (a) Neuro-module $\mathcal{G}_{\mathcal{O}} \Leftrightarrow \mathcal{L}$ resulting from the restrictive module fusion and (b) neuro-module $\mathcal{G}_{\mathcal{O} \leftrightarrow \mathcal{L}}$ resulting from the semi-restrictive module fusion and their resulting light seeking behavior. The grey color indicates the unchanged elements.

semi-restrictive $\left(\mathcal{G}_{\mathcal{O} \leftrightarrow \mathcal{L}}\right)$ evolved by our fusion method. Considering the structure of module $\mathcal{G}_{\mathcal{O}} \Leftrightarrow \mathcal{L}$ (Fig. 3 (a)) the evolved coupling between the two basic modules $\mathcal{G}_{\mathcal{O}}$ and $\mathcal{G}_{\mathcal{L}}$ does not include any new hidden neurons but a lot of new synaptic connections. These connections show many recurrences, like self-connections and rings. Nevertheless, only one 2-ring $\left(H_{3}\right.$ and $\left.O_{1}\right)$ has a critical weight parameter configuration, that provides non-trivial dynamical properties. The 2-ring between $H_{3}$ and $O_{1}$ is odd and can generate periodic as well as chaotic attractors. With respect to the resulting robot behavior (see Fig. 3 (a)) one can observe 
a strong drive to the left, if no obstacle and light is detected. In the case of obstacle detection the module produces large turning angles to avoid a collision. Again, module $\mathcal{G}_{\mathcal{O} \Leftrightarrow \mathcal{L}}$ successfully produces a light seeking behavior including the escapes from dead-ends as well as a halt in front of a light source.

The semi-restrictive evolved coupling of module $\mathcal{G}_{\mathcal{O} \leftrightarrow \mathcal{L}}$ (Fig. 3 (b)) consists of only a few new connections. There are even no new recurrences. New dynamical properties originally generated by these new connections can not be expected. They can at most provide non-trivial dynamical features which are generated by the structures of the basic modules $\mathcal{G}_{\mathcal{O}}$ and $\mathcal{G}_{\mathcal{L}}$. And again, these basic modules have strikingly changed. The self-connection of hidden neuron $H_{4}$ has become critical. Hence, $H_{4}$ can generate period-2 oscillations. Similar to the semi-restrictive extended module $\mathcal{G}_{\mathcal{O} \rightarrow \mathcal{L}}$ the former even 2-ring of basic module $\mathcal{G}_{\mathcal{O}}$ has become an odd 2-ring. Therefore, this 2-ring between $H_{1}$ and $H_{2}$ can also generate periodic as well as chaotic oscillations. A robot controlled by module $\mathcal{G}_{\mathcal{O} \leftrightarrow \mathcal{L}}$ moves straight forward if no obstacle and light is detected. It also produces a halt in front of a light source. But the turning angles generated by this module during obstacle avoidance are very large. Note, that the generation of these large turning angles reduces the exploration capabilities, insofar as we understand and define well exploration by the robot's visited area.

\subsection{Free and Starting from Scratch}

The following two light seeking modules (Fig. 4) are evolved in such a way that either the underlying initial structures can be removed or no initial structure was given.

Neuro-module $\mathcal{G}_{\mathcal{O}-\mathcal{L}}$ (Fig. 目 (a)) is an example of a free expansion. That means, although the evolutionary process was initialized with the basic module $\mathcal{G}_{\mathcal{O}}$, the elements of this initial structure were not locked during evolution. Hence, all elements of the initial structure could be modified by the variation operator during the evolutionary process, including the deletion of initialized connections. As it can be seen in Fig. 世(a) the resulting structure is purely feedforward organized. All recurrences of the initial structure $\mathcal{G}_{\mathcal{O}}$ were removed during the evolutionary process. According to this feedforward structure the resulting robot behavior is determined only by fixpoint attractors. Nevertheless, also this simply feedforward structure enables the robot to escape from deadends, to stop in front of a light source and to robustly move straight forward, if no obstacle and light is detected.

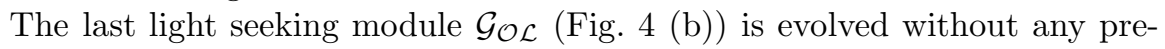
defined control, because the evolutionary process was initialized with the empty initial structure. With respect to the number of synapses and hidden neurons this is the smallest control structure and also purely feedforward organized. Although its resulting behavior shows a strong drive to the left it successfully solves the light seeking task. 
(a)

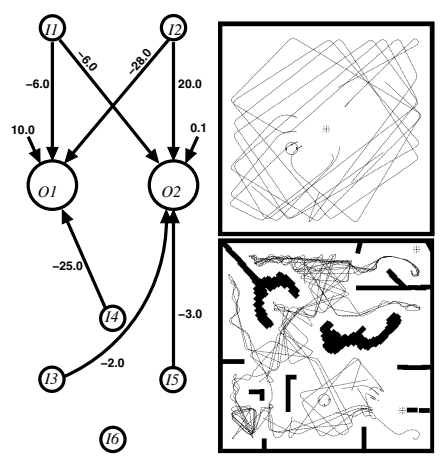

(b)

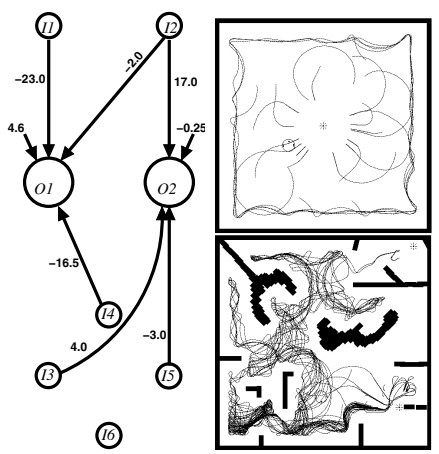

Fig. 4. (a) Neuro-module $\mathcal{G}_{\mathcal{O}-\mathcal{L}}$ resulting from the free module expansion and (b) neuro-module $\mathcal{G}_{\mathcal{O} \mathcal{L}}$ resulting from evolution starting with an empty initial structure

\section{Discussion of the Structure-Function Relations}

Clarifying the relationship between evolved structure, its inherent dynamics, and the resulting robot behavior we identify five different behavioral patterns: (1) moving forward, (2) avoiding obstacles, (3) orientation to the light, (4) halt in front of an light source, and (5) solving the conflict between obstacle avoidance and approaching the light. In the following we will only focus on two behavioral patterns: orientation to the light and halt in front of a light source.

These patterns correspond to specific sensor value configurations. The halt in front of a light source is basically characterized by a high activation of all front light sensor values, while distance sensors and the light sensor at the rear have low activations $\left(i_{3,4,5} \uparrow i_{1,2,6} \downarrow\right)$. The orientation to a light source can be characterized as the transition from behavioral pattern moving forward $\left(i_{1,2, \ldots 6} \downarrow\right)$ to the halt in front of a light. We symbolize this transition as follows: $i_{4} \uparrow i_{3,5} \uparrow i_{1,2,6} \downarrow$.

To identify relevant attractors for specific parameter configurations the four neuro-modules were simulated as dynamical systems, de-coupled from constraints of the body and environmental interactions. Based on this simulations we have an indication which attractor generates the observed behavior patterns. The results are summarized in Table 1 .

Due to its feedforward organization, the behavior relevant dynamics of neuromodules $\mathcal{G}_{\mathcal{O}} \mathcal{L}$ and $\mathcal{G}_{\mathcal{O}-\mathcal{L}}$ are purely based on fixpoint attractors. With respect to these attractors the two modules can be seen as a simple superposition of the two basic modules $\mathcal{G}_{\mathcal{O}}$ and $\mathcal{G}_{\mathcal{L}}$.

Contrary, all modules resulting from the expansion and fusion experiments show an increase of complexity according to the behavior relevant dynamical properties. This becomes most obvious, if the dynamical properties providing the orientation to the light are investigated. The bifurcation diagrams in Fig. 5 indicate that the dynamical features generating an orientation to the light are beyond simple fixpoint dynamics. 
Table 1. Attractors of the neuro-modules under specific parameter configurations

\begin{tabular}{|l|c|c|c|c|}
\hline modules & moving forward & obstacle avoidance & $\begin{array}{c}\text { halt in front } \\
\text { of a light source } \\
i_{3,4,5} \uparrow \\
i_{1,2,6} \downarrow\end{array}$ & $\begin{array}{c}\text { orienting } \\
\text { to the light } \\
i_{4} \uparrow i_{3,5} \uparrow \\
i_{1,2,6} \downarrow\end{array}$ \\
\hline \hline $\mathcal{G}_{\mathcal{O}} \downarrow$ & fixpoint & fixpoint & - & - \\
\hline $\mathcal{G}_{\mathcal{L}}$ & fixpoint & - & fixpoint & fixpoint \\
\hline \hline $\mathcal{G}_{\mathcal{O} \Rightarrow \mathcal{L}}$ & fixpoint & fixpoint & fixpoint & hysteresis \\
\hline $\mathcal{G}_{\mathcal{O} \rightarrow \mathcal{L}}$ & chaotic & fixpoint & fixpoint & chaotic \\
\hline $\mathcal{G}_{\mathcal{O} \Leftrightarrow \mathcal{L}}$ & chaotic & chaotic & fixpoint & chaotic \\
\hline $\mathcal{G}_{\mathcal{O} \leftrightarrow \mathcal{L}}$ & perio- 2 & hysteresis and period-2 & fixpoint & period-2 \\
\hline \hline $\mathcal{G}_{\mathcal{O}-\mathcal{L}}$ & fixpoint & fixpoint & fixpoint & fixpoint \\
\hline $\mathcal{G}_{\mathcal{O} \mathcal{L}}$ & fixpoint & fixpoint & fixpoint & fixpoint \\
\hline
\end{tabular}

$\mathcal{G}_{\mathcal{O} \Rightarrow \mathcal{L}}$
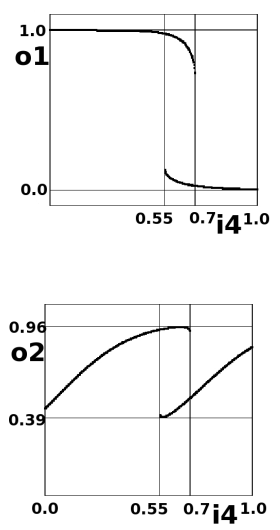

$\mathcal{G}_{\mathcal{O} \rightarrow \mathcal{L}}$
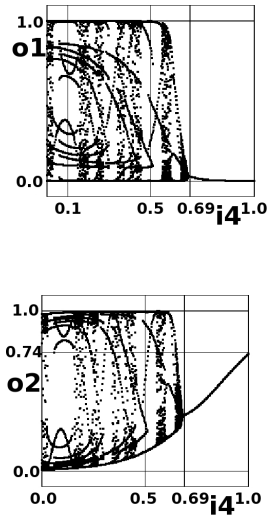

$\mathcal{G}_{\mathcal{O}} \Leftrightarrow \mathcal{L}$
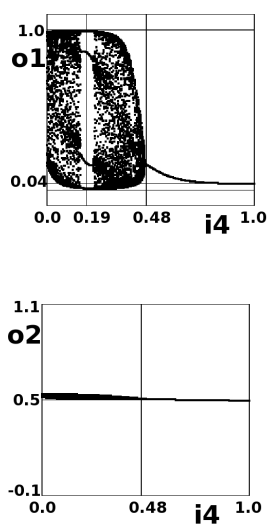

$\mathcal{G}_{\mathcal{O} \leftrightarrow \mathcal{L}}$
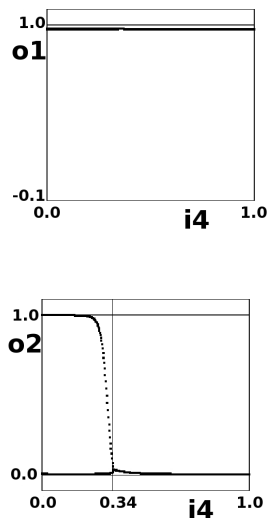

Fig. 5. Behavior relevant attractors causing a halt in front of a light source indicated by bifurcation diagrams of the four light seeking modules. Upper: output value $o_{1}$ over the input value $i_{4}$, other input values $i_{n}=0$. Lower: output value $o_{2}$ over input value $i_{4}$, other input values $i_{n}=0$.

For instance while a robot, controlled by neuro-module $\mathcal{G}_{\mathcal{O} \rightarrow \mathcal{L}}$ or $\mathcal{G}_{\mathcal{O}} \Leftrightarrow \mathcal{L}$, is approaching a light source the speed control is realized by chaotic attractors. The turning angle in module $\mathcal{G}_{\mathcal{O} \rightarrow \mathcal{L}}$ is also modulated by a chaotic attractor (Fig. [5).

Considering module $\mathcal{G}_{\mathcal{O}} \leftrightarrow \mathcal{L}$ we observe that orientation to a light source is provided by a period-2 attractor (Fig. 5). The period-2 oscillation creates a permanent alteration of $o_{2}$ between 0 and 1 . Over time this generates an effective motor signal of 0.5 , which is the mean of this stream of output values. The value 0.5 represents a turning value of zero and the robot moves straight due to $o_{1} \approx 1.0$. 

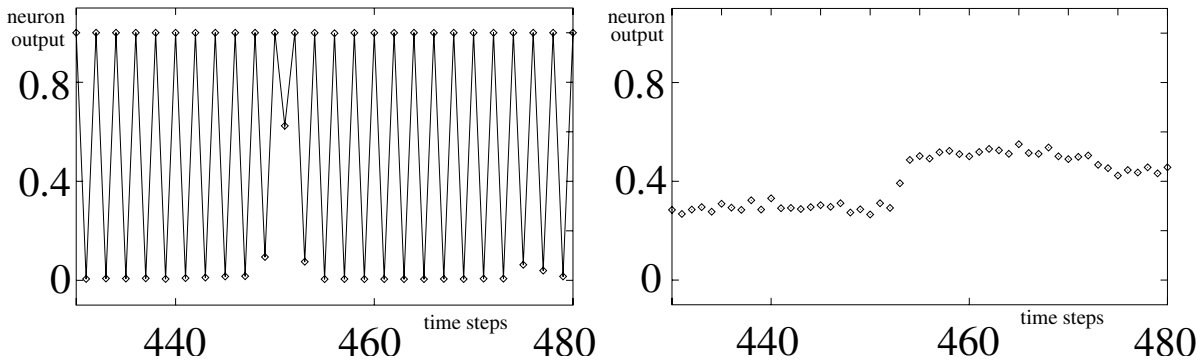

Fig. 6. The neuron outputs $o_{2}$ (left) and $i_{4}$ (right) over time of neuro-module $\mathcal{G}_{\mathcal{O}} \leftrightarrow \mathcal{L}$. The data are recorded while the robot is approaching a light source. The orientation to the light is realized by a modulation of the amplitude of a period- 2 oscillation.

If the amplitude is changing, the mean of this output stream is changing, too (between time step 440 and 460 in the left diagram of Fig. 6). Therefore, the effective motor signals become unequal zero, which creates a turn towards the light, indicated by the increased activity of $i_{4}$ (right diagram Fig. [6).

Regarding neuro-module $\mathcal{G}_{\mathcal{O} \Rightarrow \mathcal{L}}$ there is a hysteresis effect active while the robot is approaching the light. This hysteresis effect creates a discrete switch between the turning angles represented by the values 0.39 and 0.96 of $o_{2}$ (Fig. (5)). Such a hard switch produces the zigzag close to a light source (compare to path plot of Fig. 2 (a)).

\section{Conclusion}

In this paper we presented incrementally evolved neuro-modules solving a light seeking task for a Khepera robot. We have systematically applied two methods: expansion and fusion. These two methods based on a structure evolution of various recurrent neural networks. The expansion method extends the structure of RNNs, while fusion couples two RNNs to combine different behavioral functionalities. The scope of these experiment was (1) the study of the coordination of different behaviors within one neural control structure and (2) the control principles which allow the integration of new behavioral capabilities without losing the old functionality.

The extended and coupled neuro-modules show an increase of complexity with respect to the behavior relevant dynamical properties. Additionally, we have shown that if relevant dynamics of a behavioral function are modified, the control principles of this robot behavior also fundamentally change. For instance, neuro-module $\mathcal{G}_{\mathcal{O} \leftrightarrow \mathcal{L}}$ utilizes a "frequency and amplitude" coding to generate the required motor signals. Such a coding was not grounded in the basic modules, neither in $\mathcal{G}_{\mathcal{O}}$ nor in $\mathcal{G}_{\mathcal{L}}$. As simple as the presented evolution experiments are they provide a minimal setup which allowed us a detailed study of the effects of the presented incremental evolution on the dynamical properties of the resulting 
control structures which leads us to the conclusion that in ER the role of nonlinear control principles can hardly be overemphasized.

Indeed, the two examples $\mathcal{G}_{\mathcal{O}-\mathcal{L}}$ and $\mathcal{G}_{\mathcal{O} \mathcal{L}}$ demonstrate how the development of multifunctionality can be organized by a simple superposition of behavior relevant dynamical properties. Hence, multifunctionality can also be generated without new non-trivial dynamical features and non-linear couplings. But, these two examples also show: The development of a simple superposition either goes hand in hand with a remove of initial elements and functionality or has to start with an empty initial structure. These observations suggest that multifunctionality organized by linear control structures must start from scratch each time a new function has to be integrated. This will become unwieldy for open-ended evolutionary processes at a certain level of desired behavioral complexity. If those effects can already be observed in a simple combination of a positive and negative tropism, a stick to linear control structures in ER models must be carefully evaluated.

Relating our results to some state of the art research, we stress three major points: (1) Multifunctionality and task related switchings are natural properties of non-linear coupled systems [7. (2) The development of multifunctionality is a indispensable prerequisite for open-ended artificial evolutionary processes [5]. (3) Open-ended artificial evolution must develop multifunctionality incrementally to cope the scalability problem [1]3. Considering these aspects and our results, we claim, that control structures must be based on non-linear principles, if they should provide open-ended artificial evolutionary processes in Evolutionary Robotics.

\section{References}

1. Beer, R. D.: An dynamical systems perspective on agent-environment interaction. Artificial Intelligence 72 (1995) 173 - 215

2. Beer, R. D.: The dynamics of active categorical perception in an evolved model agent. Adaptive Behavior 11 (2003) 209 - 243

3. Brooks, R. A.: Artificial life and real robots. In: Proceedings of the First European Conference on Artificial Life, MIT Press (1992) 3-10

4. Clark, A.: Being There: Putting Brain, Body and World Together Again. MIT Press, 1997.

5. Bianco, R., Nolfi, S.: Toward open-ended evolutionary robotics: Evolving elemantary robotic units able to self-assemble and selfreproduce. Connection Science 16 (2004) 227-248

6. Hülse, M., Wischmann, S., and Pasemann, F.: Structure and function of evolved neuro-controllers for autonomous robots. Connection Science 16 (2004) 249-266

7. Kelso, S.: Dynamic Patterns, MIT Press, 1995.

8. Michel, O.: Khepera Simulator, Package version 2.0. Freeware mobile robot simulator written at the University of Nice Sophia-Antipolis by Oliver Michel. Downloadable from the World Wide Web at http://wwwi3s.unice.fr/ om/khep-sim.html.

9. Mondada, F., Franzi, E., Ienne, P.: Mobile robots miniturization: A tool for investigation in control algorithms. In: Proc. of ISER' 93, Kyoto, 1993. 
10. Nolfi, S., and Floreano, D.: Evolutionary Robotics: The Biology, Intelligence, and Technology of Self-Organizing Machines. MIT Press, Cambridge, 2000.

11. Pasemann, F.: Characteristics of periodic attractors in neural ring networks. Neural Networks 8 (1995) $421-429$.

12. Pasemann, F.: Structure and dynamics of recurrent neuro-modules. Theory in Biosciences 117 (1998) $1-17$.

13. Pasemann, F., Steinmetz, U., Hülse, M., and Lara, B.: Robot control and the evolution of modular neurodynamics. Theory in Biosciences 120 (2001) 311-326

14. Walker, J., Garrett, S., Wilson, M.: Evolving controllers for real robots: A survey of the literature. Adaptive Behavior 11 (2003) 179-203. 